PROCEEDINGS OF THE

AMERICAN MATHEMATICAL SOCIETY

Volume 127, Number 11, Pages 3169-3174

S 0002-9939(99)04910-2

Article electronically published on May 13, 1999

\title{
INFINITE HOMOGENEOUS ALGEBRAS ARE ANTICOMMUTATIVE
}

\author{
DRAGOMIR Ž. ĐOKOVIĆ AND LOWELL G. SWEET
}

(Communicated by Lance W. Small)

\begin{abstract}
A (non-associative) algebra $A$, over a field $k$, is called homogeneous if its automorphism group permutes transitively the one dimensional subspaces of $A$. Suppose $A$ is a nontrivial finite dimensional homogeneous algebra over an infinite field. Then we prove that $x^{2}=0$ for all $x$ in $A$, and so $x y=-y x$ for all $x, y \in A$.
\end{abstract}

\section{INTRODUCTION}

The algebras to be discussed are assumed to be finite dimensional over a field $k$ and not necessarily associative. Thus the multiplication $A \times A \rightarrow A$ can be an arbitrary bilinear map. Such an algebra $A$ is nontrivial if $\operatorname{dim} A>1$ and $A^{2} \neq 0$. Also, $\operatorname{Aut}(A)$ denotes the group of algebra automorphisms of $A$.

An algebra $A$ is said to be extremely homogeneous if $\operatorname{Aut}(A)$ acts transitively on $A \backslash\{0\}$. The concept of an extremely homogeneous algebra arose from a particular problem in the structure of certain finite p-groups as studied by Boen, Rothaus, and Thompson [2]. Extremely homogeneous algebras were also investigated by Kostrikin [6].

An algebra $A$ is homogeneous if $\operatorname{Aut}(A)$ permutes transitively the one dimensional subspaces of $A$. Homogeneous algebras over finite fields were studied by Shult [11] and Gross [4]. The classification of homogeneous algebras over finite fields was completed by Ivanov [5]. He showed that if $A$ is a nontrivial homogeneous algebra over a finite field $k$, then $k=\mathrm{GF}(2)$ and $A$ is of a type previously described by Kostrikin.

The condition of homogeneity is very strong indeed. For instance, if $k$ is algebraically closed, then the second author [10] has shown that there are no nontrivial homogeneous algebras. When $k$ is the real field, the first author [3] has classified all homogeneous algebras. In that case there are up to isomorphism exactly three nontrivial homogeneous algebras. Also, MacDougall and Sweet [7], [8], [9] have found all homogeneous algebras of dimension 2,3, or 4 with no restrictions on the ground field.

If $a \in A$, we denote by $\langle a\rangle$ the subalgebra of $A$ generated by $a$. If $A$ is homogeneous, then $\langle a\rangle$ is also homogeneous and it is easy to show that $\langle a\rangle=\langle x\rangle$ for any

Received by the editors January 7, 1998 and, in revised form, February 6, 1998.

1991 Mathematics Subject Classification. Primary 17D99; Secondary 17A36, 15A69.

Key words and phrases. Non-associative algebras, automorphism group, hypersurface.

This work was supported in part by the NSERC Grant A-5285.

(C)1999 American Mathematical Society 
$x \in\langle a\rangle \backslash\{0\}$. Indeed if $x \in\langle a\rangle \backslash\{0\}$, there is some $\varphi \in \operatorname{Aut}(A)$ taking $k \cdot a$ to $k \cdot x$, hence carrying $\langle a\rangle$ to $\langle x\rangle$, so $\varphi$ yields an automorphism of $A$. It follows that if $A$ is homogeneous and $a \in A$, then $\langle a\rangle$ is an algebra with no proper subalgebras. Algebras with no proper subalgebras were investigated by Artamonov [1]. In particular he showed that if $A$ is a nontrivial algebra with no proper subalgebras and $\operatorname{dim} A$ is a prime, then $\operatorname{Aut}(A)$ is a finite group.

The purpose of this paper is to show that if $A$ is a homogeneous algebra over an infinite field, then $x^{2}=0$ for all $x \in A$. This of course implies that $A$ is anticommutative. It also implies that if $A$ is a nontrivial homogeneous algebra with no proper subalgebras, then the underlying field $k$ must be finite (and so these algebras are known, they must be of Kostrikin type).

In the remainder of the paper we use the following notation: $A$ is a homogeneous algebra over an infinite field $k, K$ is an algebraic closure of $k, A_{K}=K \otimes_{k} A$ is the $K$-algebra obtained from $A$ by extension of scalars, and $G_{k}$ (resp. $G_{K}$ ) is the automorphism group of the algebra $A$ (resp. $A_{K}$ ). For $x \in A_{K}, L_{x}: A_{K} \rightarrow A_{K}$ denotes left multiplication by $x$ (and similarly $R_{x}$ denotes right multiplication by $x$ ). If $A$ is an algebra, then $A^{+}$is the commutative algebra obtained from $A$ by introducing the new multiplication $x \cdot y=x y+y x$ (note that $\operatorname{Aut}(A)$ is a subgroup of $\operatorname{Aut}\left(A^{+}\right)$and so $A$ homogeneous implies $A^{+}$homogeneous). By $A^{\text {opp }}$ we denote the opposite algebra of $A$ in which $x \cdot y=y x$. As usual we set $k^{*}=k \backslash\{0\}$ and $K^{*}=K \backslash\{0\}$.

We thank the referee for his suggestions.

\section{RESULTS AND PROOFS}

The following three lemmas are needed for the proof of the main theorem.

Lemma 1. Let $A$ be a nontrivial homogeneous algebra over an infinite field $k$. If $a^{2} \in k \cdot a, a \in A$, then $a^{2}=0$.

Proof. This result follows directly from Theorem 7 of [8].

Lemma 2. Let $A=\left\langle a_{0}\right\rangle$ be a nontrivial homogeneous algebra over an infinite field $k$. If $a \in A \backslash\{0\}$, then the stabilizer $H \subset G_{K}$ of the 1-dimensional subspace $K \cdot a$ is trivial.

Proof. Assume that $H$ contains an element $h \neq 1$. Then $h(a)=\lambda a$ for some $\lambda \in K^{*}, \lambda \neq 1$. We define $a^{2^{r}}, r \geq 0$, inductively by $a^{1}=a, a^{2^{r+1}}=\left(a^{2^{r}}\right)^{2}$. Since $A=\left\langle a_{0}\right\rangle$ has dimension $>1$, we must have $a_{0}^{2} \neq 0$, hence by homogeneity all nonzero elements of $A$ have nonzero square, hence $a^{2^{r}} \neq 0$ for all $r \geq 0$ and $h\left(a^{2^{r}}\right)=\lambda^{2^{r}} a^{2^{r}}$. As $h$ has only finitely many eigenvalues, $\lambda$ is a root of unity, say, $\lambda^{d}=1, d>1$.

As $A=\langle a\rangle, A$ has a basis $\left\{b_{1}, b_{2}, \ldots, b_{n}\right\}$ where each $b_{i}$ is a product of $n_{i}$ a's under some bracketing. Let $m$ be a positive integer such that $t=d m>n_{i}$ for all $i$. Then $h\left(a^{t}\right)=\lambda^{t} a^{t}=a^{t}$, where $a^{t}$ is any product of $t a$ 's. If $a^{t} \neq 0$ for some bracketing, then $A=\left\langle a^{t}\right\rangle$ which implies that $h=1$, a contradiction. Hence we have $a^{t}=0$ for all bracketing. But then $L_{a}^{t-n_{i}}\left(b_{i}\right)=0$, and so $L_{a}^{t}\left(b_{i}\right)=0$ for all $i$. Thus $L_{a}^{t}=0$, and similarly $R_{a}^{t}=0$. Hence $A$ is a special nil algebra as defined in [10]. Since $k$ is infinite, it follows from Theorem 2 of the above paper that $A^{2}=0$, which is a contradiction. 
Lemma 3. Let $A=\left\langle a_{0}\right\rangle$ be a nontrivial commutative homogeneous algebra over an infinite field $k$ where char $k \neq 2$. Then $A$ has no zero divisors.

Proof. Suppose $a b=0$ for some $a, b \in A \backslash\{0\}$. Since $A=\langle a\rangle$ and $\operatorname{dim} A>1$, we have $a^{2} \neq 0$ and so $a$ and $b$ are linearly independent. Hence there exist $g_{1}, g_{2} \in G_{k}$ such that

$$
g_{1}(a)=\lambda(a+b), \quad g_{2}(a)=\mu(a-b), \quad \lambda, \mu \in k^{*} .
$$

But then, using $a b=b a=0$,

$$
g_{1}\left(a^{2}\right)=\lambda^{2}\left(a^{2}+b^{2}\right), \quad g_{2}\left(a^{2}\right)=\mu^{2}\left(a^{2}+b^{2}\right)
$$

and so $g_{2}^{-1} g_{1}$ is in the stabilizer of $k \cdot a^{2}$. Lemma 2 implies that $g_{1}=g_{2}$ and (1) gives a contradiction.

We can now prove our main result.

Theorem. Let $A$ be a nontrivial homogeneous algebra over an infinite field $k$. Then $x^{2}=0$ for all $x \in A$.

Proof. Assume that $x^{2} \neq 0$ for some $x \in A$. Since $A$ is homogeneous, this implies that $x^{2} \neq 0$ for all nonzero $x \in A$. Our goal is to obtain a contradiction. If char $k \neq 2$, by replacing $A$ with $A^{+}$, we may assume that $A$ is commutative.

Let us fix a nonzero element $b \in A$. By Lemma $1, b^{2} \notin k \cdot b$. By replacing $A$ with the subalgebra generated by $b$, we may assume that $A=\langle b\rangle$. Suppose $L_{b}$ is nilpotent. If char $k \neq 2$, then $R_{b}=L_{b}$ is also nilpotent and so $A$ is a special nilalgebra. As noted in the proof of Lemma 2, this is impossible. If char $k=2$, we obtain the same contradiction if $L_{b}$ and $R_{b}$ are both nilpotent. So, if necessary, by replacing $A$ by $A^{\text {opp }}$, we can assume that $L_{b}$ is not nilpotent.

Let us fix a nonzero eigenvalue $\mu \in K$ of $L_{b}$ and set $a:=\mu^{-1} b \in A_{K}$. Let $P: A_{K} \rightarrow K$ be the polynomial function defined by

$$
P(x):=\operatorname{det}\left(1-L_{x}\right)
$$

and let $P=P_{1} P_{2} \cdots P_{m}$ be its prime factorization. Since 1 is an eigenvalue of $L_{a}$, we have $P(a)=0$. We may assume that the factors are numbered so that $P_{1}(a)=0$ and normalized so that $P_{i}(0)=1$ for all $i$. Let $H$ be the hypersurface in $A_{K}$ defined by $P(x)=0$ and let $H_{1}$ be its irreducible component defined by $P_{1}(x)=0$.

For $g \in G_{K}$ and $x \in A_{K}$ we have $g \circ L_{x} \circ g^{-1}=L_{g(x)}$ and consequently $P$ is $G_{K}$ invariant. Hence the orbit $\mathcal{O}:=G_{K} \cdot a$ is contained in $H$.

Let

$$
\pi: A_{K} \backslash\{0\} \rightarrow \mathbf{P}\left(A_{K}\right)
$$

be the canonical projection onto the projective space $\mathbf{P}\left(A_{K}\right)$. The image of $A \backslash\{0\}$ under $\pi$ will be identified with the projective space $\mathbf{P}(A)$ whose points are the 1-dimensional $k$-subspaces of $A$. We have

$$
\mathbf{P}(A)=\pi\left(G_{k} \cdot b\right) \subset \pi(\mathcal{O}) \subset \mathbf{P}\left(A_{K}\right) .
$$

Since $k$ is infinite, $\mathbf{P}(A)$ is Zariski dense in $\mathbf{P}\left(A_{K}\right)$. Hence $\operatorname{dim} \mathcal{O} \geq n-1$, and since $\mathcal{O} \subset H$, we infer that $\operatorname{dim} \mathcal{O}=n-1$.

We claim that the affine algebraic group $G_{K}$ is connected (in the Zariski topology). Indeed let $G_{K}^{0}$ be the identity component of $G_{K}$ and let $g \in G_{K}$ be arbitrary. 
Both $\pi\left(G_{K}^{0} \cdot a\right)$ and $\pi\left(g G_{K}^{0} \cdot a\right)$ are nonempty open subsets of $\mathbf{P}\left(A_{K}\right)$. Hence they have nontrivial intersection, i.e., there exist $g_{1}, g_{2} \in G_{K}^{0}$ such that $g g_{1} \cdot K a=g_{2} \cdot K a$. By Lemma 2 we conclude that $g g_{1}=g_{2}$ and so $g=g_{2} g_{1}^{-1} \in G_{K}^{0}$ and our claim is verified.

It follows that each factor $P_{i}$ of the $G_{K}$-invariant polynomial $P$ is $G_{K}$-invariant. Hence $H_{1}$ is $G_{K}$-invariant and so $\mathcal{O} \subset H_{1}$ and $\mathcal{O}$ is open and dense in $H_{1}$. Let $d$ be the degree of $P_{1}$ and write $P_{1}=P_{10}+P_{11}+\cdots+P_{1 d}$ where $P_{1 i}$ is a homogeneous polynomial of degree $i$. We set $f=-P_{1 d}$.

We claim that $d>1$. Assume that $d=1$. Then $H_{1}$ is the hyperplane $f(x)=1$; hence $f(a)=1$ and $f(b)=\mu$. If $g \in G_{k}$ and $g \neq 1$, then also $f(g \cdot b)=\mu$. Thus $c=b-g \cdot b$ is a nonzero vector of $A$ such that $f(c)=0$ contradicting the homogeneity. Hence our claim is proved.

Since $k$ is infinite and $f \neq 0, f$ cannot vanish identically on $\mathbf{P}(A)$. As $\pi\left(G_{k} \cdot b\right)=$ $\mathbf{P}(A)$, it follows that $f(b) \neq 0$, and so $f(a) \neq 0$. We conclude that the polynomial $P_{1}(t a)$ in the variable $t$ must also have degree $d$. But $P_{1}(a)=0$ and so $t=1$ is a root of the polynomial $P_{1}(t a)$. Let $\lambda \in K$ be an arbitrary root of that polynomial. Then $\lambda \neq 0$ and $\lambda \mathcal{O}$ and $\mathcal{O}$ are both open dense subsets of $H_{1}$. Hence $\lambda \mathcal{O} \cap \mathcal{O} \neq \emptyset$, and consequently $\lambda \mathcal{O}=\mathcal{O}$. Now Lemma 2 implies that $\lambda=1$. We conclude that $t=1$ is the only root of the polynomial $P_{1}(t a)$ and, by using $P_{1}(0)=1$, we infer that

$$
P_{1}(t a)=(1-t)^{d} .
$$

Since $P_{1}$ is $G_{K}$-invariant and $\mathcal{O}$ is dense in $H_{1}$, we have

$$
P_{1}(t x)=(1-t)^{d}, \quad \forall x \in H_{1} .
$$

This polynomial identity (in the variable $t$ ) implies that each of the polynomials

$$
P_{1 i}(x)-(-1)^{i}\left(\begin{array}{c}
d \\
i
\end{array}\right), \quad 0 \leq i \leq d,
$$

vanishes on $H_{1}$. Since $P_{1}$ is irreducible, $P_{1}$ must divide each of these polynomials. It follows that $P_{1 i}=0$ for $0<i<d$, char $k=p>0$, and

$$
\left(\begin{array}{l}
d \\
i
\end{array}\right) \equiv 0 \quad(\bmod p), \quad 0<i<d .
$$

The last condition implies that $d=p^{s}$ for some integer $s \geq 1$. Since $P_{10}=P_{1}(0)=$ 1 , we have $P_{1}=1-f$ where $f=-P_{1 d}$ is homogeneous of degree $d$.

For $x \in A_{K}$ and $1 \leq i \leq p$, we define the powers $x^{i}$ recursively by $x^{1}=x$, and $x^{i}=x \cdot x^{i-1}$ for $1<i \leq p$. Next we define the powers $x^{p^{i}}$ for $i>1$ by

$$
x^{p^{i}}=\left(x^{p^{i-1}}\right)^{p} .
$$

It follows from these definitions that for all $x \in A_{K}$ and $g \in G_{K}$ we have

$$
g\left(x^{p^{i}}\right)=g(x)^{p^{i}} .
$$

We claim that $x^{p^{i}} \neq 0$ for nonzero $x \in A$ and $i \geq 0$. If $p>2$, this follows from Lemma 3 , and for $p=2$ from our assumption that $A=\langle b\rangle$.

Let $N$ be the smallest positive integer such that

$$
a, a^{p}, a^{p^{2}}, \ldots, a^{p^{N}}
$$


are $K$-linearly dependent. Then

$$
\sum_{i=0}^{N} \alpha_{i} a^{p^{i}}=0
$$

with $\alpha_{i} \in K$ and $\alpha_{N}=1$.

Assume that $\alpha_{0}=0$. As $b=\mu a \in A \backslash\{0\}$, there exists $g \in G_{k}$ such that $g\left(a^{p}\right)=\lambda a$ for some $\lambda \in K^{*}$. Equation (2) implies that

$$
\sum_{i=1}^{N} \alpha_{i} g\left(a^{p}\right)^{p^{i-1}}=0
$$

But then

$$
\sum_{i=0}^{N-1} \alpha_{i+1} \lambda^{p^{i}} a^{p^{i}}=0
$$

and this contradicts our choice of $N$. So we have shown that $\alpha_{0} \neq 0$.

As $\mathcal{O}$ is dense in $H_{1}$, it follows from (2) that

$$
\sum_{i=0}^{N} \alpha_{i} x^{p^{i}}=0, \quad \forall x \in H_{1} .
$$

Hence each coordinate function of the polynomial map

$$
x \rightarrow \sum_{i=0}^{N} \alpha_{i} x^{p^{i}}
$$

must be divisible by $P_{1}=1-f$, and so

$$
\sum_{i=0}^{N} \alpha_{i} x^{p^{i}}=(1-f(x)) \sum_{i=1}^{p^{N}-d} Q_{i}(x)
$$

where $Q_{i}$ is a homogeneous polynomial map of degree $i$. By extracting the homogeneous components of degrees congruent to $1 \bmod p$, we obtain that

$$
\alpha_{0} x=(1-f(x)) \sum_{i \equiv 1(\bmod p)} Q_{i}(x) .
$$

It follows that $Q_{1}(x)=\alpha_{0} x \neq 0$. Hence the right hand side has degree $\geq d+1$, and we have a contradiction.

\section{REFERENCES}

1. V. A. Artamonov, On algebras without proper subalgebras, Math. USSR Sbornik 33 (1977), 375-401.

2. J. Boen, O. Rothaus and J. Thompson, Further results on p-automorphic p-groups, Pacific J. Math. 12 (1962), 817-821. MR 27:25536

3. D. Ž. Djoković, Real homogeneous algebras, Proc. Amer. Math. Soc. 41 (1973), 457-462. MR 48:11227

4. F. Gross, Finite automorphic algebras over GF(2), Proc. Amer. Math. Soc. 4 (1971), 10-14. MR 44:4063

5. D. N. Ivanov, On homogeneous algebras over GF(2), Vestnik Moskov. Univ. Matematika 37 (1982), 69-72. MR 83k:17003

6. A. I. Kostrikin, On homogeneous algebras, Izvestiya Akad. Nauk. USSR 29 (1965), 471-484. MR 31:219

7. J. A. MacDougall and L. G. Sweet, Three dimensional homogeneous algebras, Pacific J. Math. 74 (1978), 153-162. MR 57:6121 
8. L. G. Sweet and J. A. MacDougall, Four dimensional homogeneous algebras, Pacific J. Math. 129(2) (1987), 375-383. MR 89c:17003

9. L. G. Sweet, On homogeneous algebras, Pacific J. Math. 59 (1975), 585-594. MR 52:8202

10. L. G. Sweet, On the triviality of homogeneous algebras over an algebraically closed field, Proc. Amer. Math. Soc. 48 (1975), 321-324. MR 51:637

11. E. E. Shult, On the triviality of finite automorphic algebras, Illinois J. Math. 13 (1969), 654-659. MR 40:1442

Department of Pure Mathematics, University of Waterloo, Waterloo, Ontario, CANADA N2L 3G1

E-mail address: dragomir@herod.uwaterloo.ca

Department of Mathematics, University of Prince Edward Island, Charlottetown, Prince Edward Island, Canada C1A 4P3

E-mail address: sweet@upei.ca 